\title{
Colgajo de So en reconstrucción de miembro inferior
}

\section{So muscle flap in lower limb reconstruction}

\author{
Márquez Zevallos*, Alcócer Cordero, P.*, Bermúdez Farías, W.**, \\ Chalén Zambrano, S. ${ }^{* \star *}$, Navarrete Quiróz, L.****
}

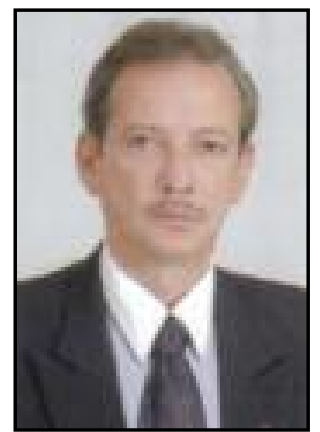

Márquez Zevallos

\section{Resumen}

El tratamiento de la extremidad inferior siempre ha sido una dura prueba para el cirujano plástico. La situación anatómica de la tibia, desprovista de protección muscular en su aspecto anterior, la hace altamente vulnerable ante cualquier traumatismo de la extremidad. Toda lesión a este nivel exige un tratamiento óseo y cutáneo adecuados, que deben realizarse primariamente y en forma conjunta. En el presente trabajo describimos 3 casos de pacientes con traumatismos de extremidad inferior, fracturas acompañadas de pérdida de sustancia, en los cuales se practicaron colgajos musculares para reconstruir las partes afectadas. Estos colgajos, en primera instancia se tomaron del músculo sóleo, pero encontramos el inconveniente del grosor exagerado que presentaba la cobertura a pesar de su atrofia posterior y del defecto estético desagradable que quedaba en la zona donante. Por todo ello realizamos una variante: en lugar de tomar la mitad del músculo como indica la técnica convencional, tomamos solo un cuadrante que sobrevivió a expensas de un solo pedículo vascular dominante. El cuadrante restante sirvió para hacer menos notorio el defecto de la zona donante. Los 3 casos tuvieron éxito y los pacientes están muy satisfechos con los resultados estéticos. Presentamos una alternativa quirúrgica innovadora de Colgajo de Sóleo que se puede aplicar satisfactoriamente para la cobertura de defectos de miembro inferior, al cual hemos llamado colgajo de So.

Palabras clave $\begin{array}{r}\text { Reconstrucción miembro inferior. Col- } \\ \text { gajo muscular. Músculo sóleo. }\end{array}$
Código numérico $4021-40212$

\begin{tabular}{|ll|}
\hline Key words & $\begin{array}{l}\text { Lower limb reconstruction. Muscular } \\
\text { flap. Soleous muscle. }\end{array}$ \\
Numeral Gode & $4021-40212$ \\
\hline
\end{tabular}

Lower limb treatment has always been a headache to the plastic surgeon. Tibial anatomy, doesn't has frontal muscle protection and it's vulnerable in any trauma. Every lesion in the lower limb must be treated by traumatologyst and plastic surgeon. In this study we present 3 patients with lower limb trauma, fractures and soft tissue lost, treated with muscle flap to cover affected parts. First, we took the flaps from soleous muscle, but we had problems with flap thickness and with the disgusting defect crated in the donor site. So we have created a variety: instead of taking half of the muscle, we took just a quarter, which survived with only one dominant vessel. The other quarter improved de defect in the donor site. The 3 patients were successful and they are happy with the results. We present an innovative surgical variant in muscle Soleous flap that can be useful in lower limb reconstruction, and that we have denominated So flap. 


\section{Introducción}

El tratamiento de las fracturas expuestas en el tercio distal de los miembros inferiores siempre ha sido un reto tanto para el traumatólogo como para el cirujano plástico, pues las deficiencias circulatorias más el trauma agregado en algunos casos o el componente metabólico en otros y la escasa cobertura de piel que tiene la tibia en su borde anterior, hacen que las lesiones a ese nivel sean de larga y dudosa evolución, más aún cuando el tratamiento no es adecuado asegurando una buena cobertura irrigada $(1,2)$.

En el año 1966 Ger realiza los primeros colgajos musculares de rotación para la cobertura de estas lesiones. Vásconez, cirujano plástico ecuatoriano de amplia trayectoria a nivel mundial, investiga y realiza en 1974 los primeros colgajos pediculados musculares irrigados con su principal arteria nutricia. Maqueira, en 1983, describe los colgajos musculares en isla.

Para realizar un buen tratamiento de una lesión, es necesario establecer primero el diagnóstico correcto de la misma y para esto, debemos basarnos en la clasificación de las heridas hecha por Gustilo en 1984 $(3,4)$. A continuación, nuestros recursos deben ir dirigidos básicamente a restablecer la estabilidad del esqueleto, la dinamización del miembro mediante la reparación inmediata de músculo-tendón-vaso-nervio (5), y finalmente la reparación de los tegumentos, ya sea mediante injertos o colgajos según las necesidades del caso.

La Cirugía Plástica ha desarrollado cada vez más modificaciones para combinar técnicas de manera que el paciente se recupere tanto funcional como estéticamente; es así, como en ciertos casos se ha dejado de lado el uso de los colgajos dermograsos y musculocutáneos, para dar paso a la aplicación de colgajos musculares (6), que tras sufrir la atrofia correspondiente, son injertados en torno a la tercera semana de evolución, obteniéndose resultados estéticos y funcionales satisfactorios en comparación con otras técnicas convencionales.

Es importante mantener dos principios básicos en la reconstrucción en Cirugía Plástica, a saber, el desbridamiento agresivo profundo y temprano, que debe establecerse en el menor tiempo posible, y del que dependerá el segundo principio que es la reconstrucción precoz de los defectos (1).

Mathes y Nahai (7) en 1982, describieron una clasificación para la reconstrucción del miembro inferior basada exclusivamente en colgajos musculares de acuerdo a los pedículos vasculares que estos poseen:

I. El pedículo vascular del músculo es uno solo y su vitalidad depende de él; así tenemos los colgajo de gemelo, recto anterior y tensor de la fascia lata.
II. Tienen un pedículo principal y dos pedículos secundarios y la supervivencia del colgajo está dada por la unión de dos de ellos; así tenemos los colgajo de bíceps femoral, flexor corto de los dedos, peróneo, semitendinoso y vasto externo.

III. Tienen dos pedículos principales, pero su vitalidad puede depender de uno solo: es el caso del glúteo mayor y del serrato mayor.

IV. Tienen varios pedículos principales y su supervivencia depende de mínimo dos de sus pedículos; es el caso del sartorio, extensor largo de los dedos, extensor largo del dedo gordo, flexor largo del dedo gordo y flexor largo de los dedos.

V. Tienen un pedículo principal y varios secundarios y su vitalidad puede depender del principal o de sus secundarios, por ejemplo, los colgajos de pectoral mayor y de dorsal ancho.

Nuestro objetivo en el presente artículo es el describir nuestra experiencia con la puesta en práctica de un colgajo muscular de sóleo para la reconstrucción del tercio distal de la pierna, pero con una variante innovadora que posteriormente explicaremos.

\section{Anatomía quirúrgica del músculo Sóleo}

El sóleo es un músculo ancho y plano que se extiende por el dorso de la pierna, inmediatamente por debajo del gemelo. Tiene su origen proximal en el tercio proximal del peroné y en la línea poplítea de la tibia y en el aspecto dorsal de ambos huesos. Distalmente, las fibras se reúnen en un tendón ancho que forma parte del tendón de Aquiles y termina en el calcáneo. Su vascularización es de tipo segmentario: recibe un pedículo vascular principal proximalmente y varios pedículos secundarios en su tercio distal, que permiten elevar cada mitad muscular basada en el pedículo correspondiente (8).

La arteria tibial posterior, acompañada del nervio tibial, sigue un trayecto descendente en la superficie profunda del músculo sóleo, y da de 3 a 5 ramas vasculares para la mitad muscular distal. Conservando las dos ramas distales, que se localizan 5 y $7 \mathrm{~cm}$. por encima del maléolo medial, es posible rotar la mitad muscular distal con completa viabilidad.

La inervación motora del sóleo viene dada por ramas del nervio tibial posterior, que entran en la superficie muscular profunda a nivel de su tercio proximal, junto al pedículo vascular superior.

La función del sóleo consiste, junto al gemelo, en la flexión plantar del pie e interviene especialmente en la marcha y en la carrera. El empleo de este músculo como colgajo, con la conservación completa de los gemelos, no acarrea alteración funcional importante, de modo que es un músculo utilizable como colgajo. 

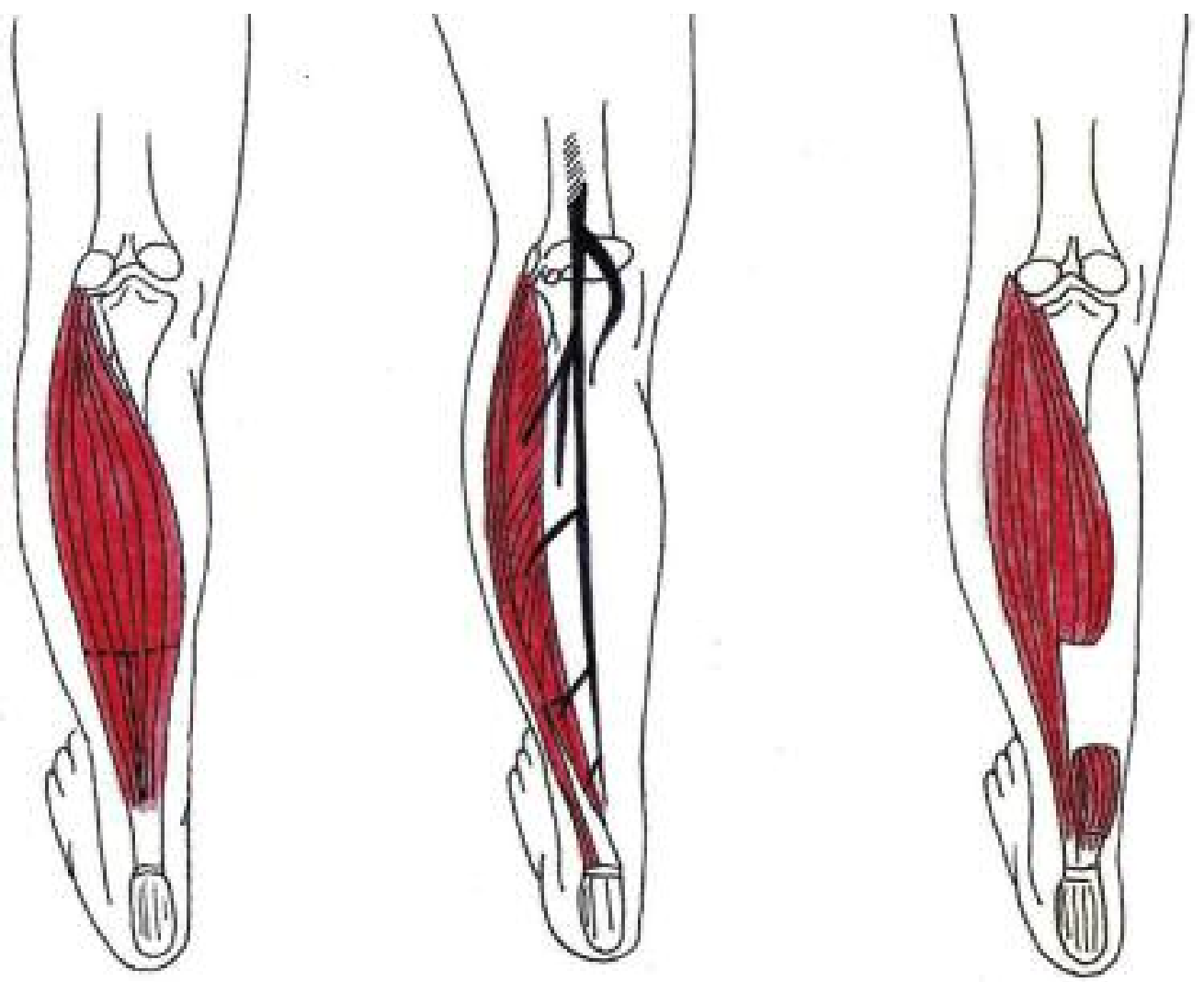

Fig. 1. Esquema del músculo soleo, en el que se aprecian los pedículos vasculares y el sitio de sección del músculo para su rotación.

Material y método

Empleamos colgajos de músculo sóleo con una variante de la técnica convencional en 3 pacientes con traumatismo de miembro inferior que presentaron fracturas óseas y pérdida de sustancia.

\section{Técnica quirúrgica}

La intervención se realizó en todos los casos con el paciente en decúbito ventral y bajo anestesia general.

Practicamos una incisión medial paralela al eje longitudinal que va desde la flexura poplítea hasta $5 \mathrm{~cm}$. por encima del talón. Dicha incisión se profundizó con electrobisturí hasta alcanzar y abrir la fascia de los gemelos. Los gemelos se separan para disecar en forma roma el sóleo (Fig.1).

La elevación de la parte proximal del músculo sóleo con separación superficial es relativamente sencilla: desde el tendón de Aquiles se separa con bisturí el extremo distal y se eleva en sentido proximal, ligando los pedículos vasculares distales que vienen de la tibial posterior. El la disección profunda debemos ser más cuidadosos y proteger la arteria tibial posterior y el nervio tibial en todo su recorrido (9-10).

La elevación de la mitad distal de este músculo se efectúa igualmente a través de un abordaje medial. La disección de las caras superficial y profunda se reali- za separándolo desde el tendón de Aquiles en idénticas condiciones a las ya descritas.

Los bordes musculares se separan de la tibia y del peroné y el cuerpo muscular se secciona en su mitad, ya que el colgajo, en su técnica convencional, solamente incluye la mitad muscular distal. Debemos conservar por lo menos dos de los pedículos, ya sean superiores o inferiores para la supervivencia de los colgajos.

Nuestra variante consiste en utilizar, en vez de la mitad superior o inferior, sólo un cuarto del sóleo, que según el caso puede ser el derecho o el izquierdo, superior o inferior, conservando sólo un pedículo vascular debido al ángulo de rotación que tuvimos que aplicar en nuestros casos y, aunque la viabilidad de la mitad distal según la literatura es menor que la de la mitad proximal, la hemos utilizado con éxito en los casos que presentaremos.

Una vez fijado el colgajo al defecto con Vicryl ${ }^{\circledR} 2$ 0 mediante puntos simples, cerramos la fascia y la piel con Ethilón ${ }^{\circledR}$ 3-0 en puntos de colchonero hasta llegar a $2 \mathrm{~cm}$. por encima del pedículo muscular. Cubrimos con vendajes estériles.

Durante 3 semanas revisamos la viabilidad del colgajo, y al finalizar la tercera semana realizamos un injerto libre de piel sobre el colgajo, que es abierto a los 4 días. 

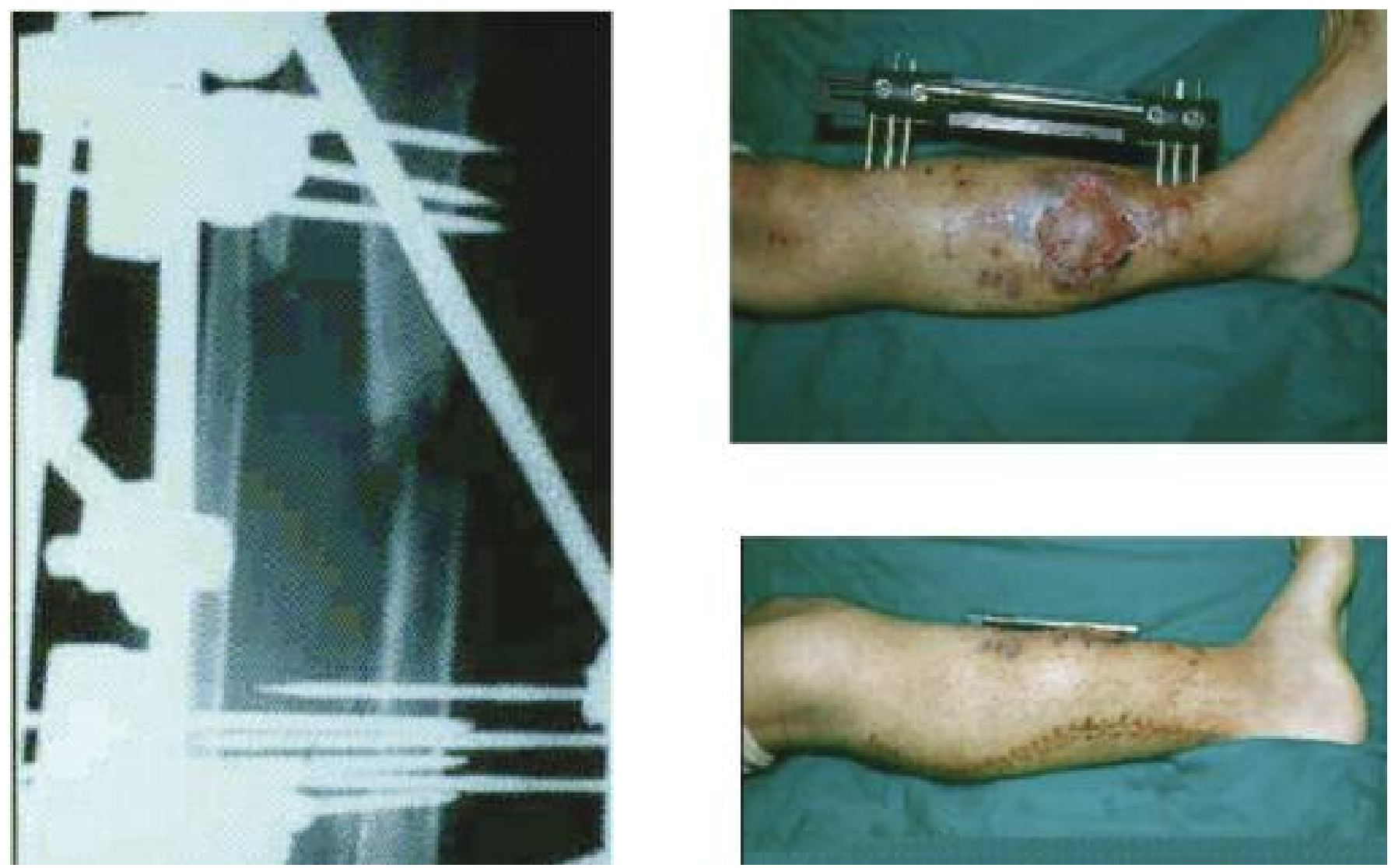

Fig. 2. Fractura de tercio medio de tibia y peroné grado II de Gustilo. Se realizó Colgajo de So, utilizando el cuadrante superior interno del sóleo, tunelizado y fijado en la región anterior, cerrando completamente el defecto.
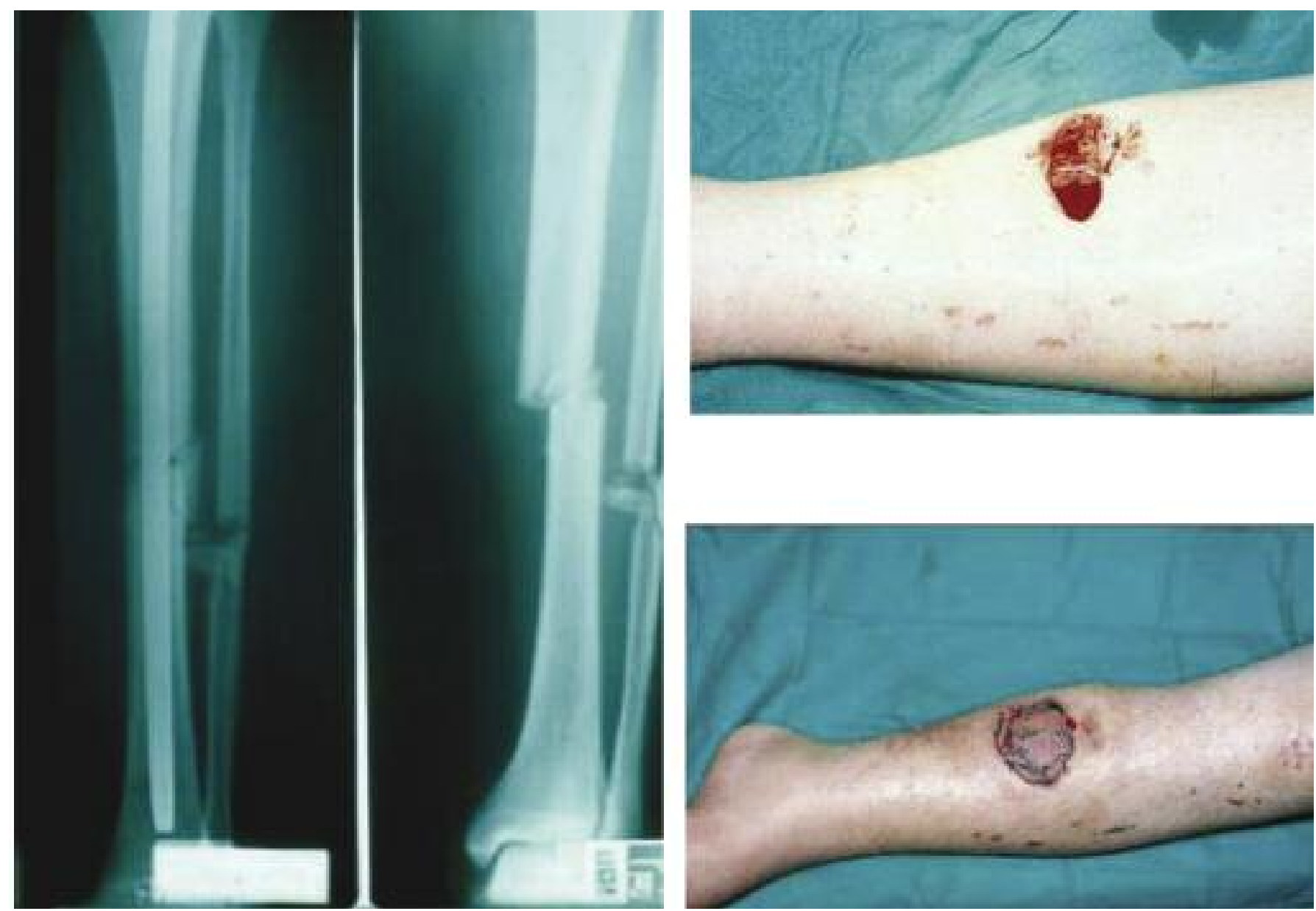

Fig. 3. Fractura de tercio inferior de tibia y peroné grado II de Gustilo. Se realizó Colgajo de So, utilizando el cuadrante inferior interno de sóleo, tunelizado y fijado al defecto. Tres semanas después se realizó injerto libre de piel. 

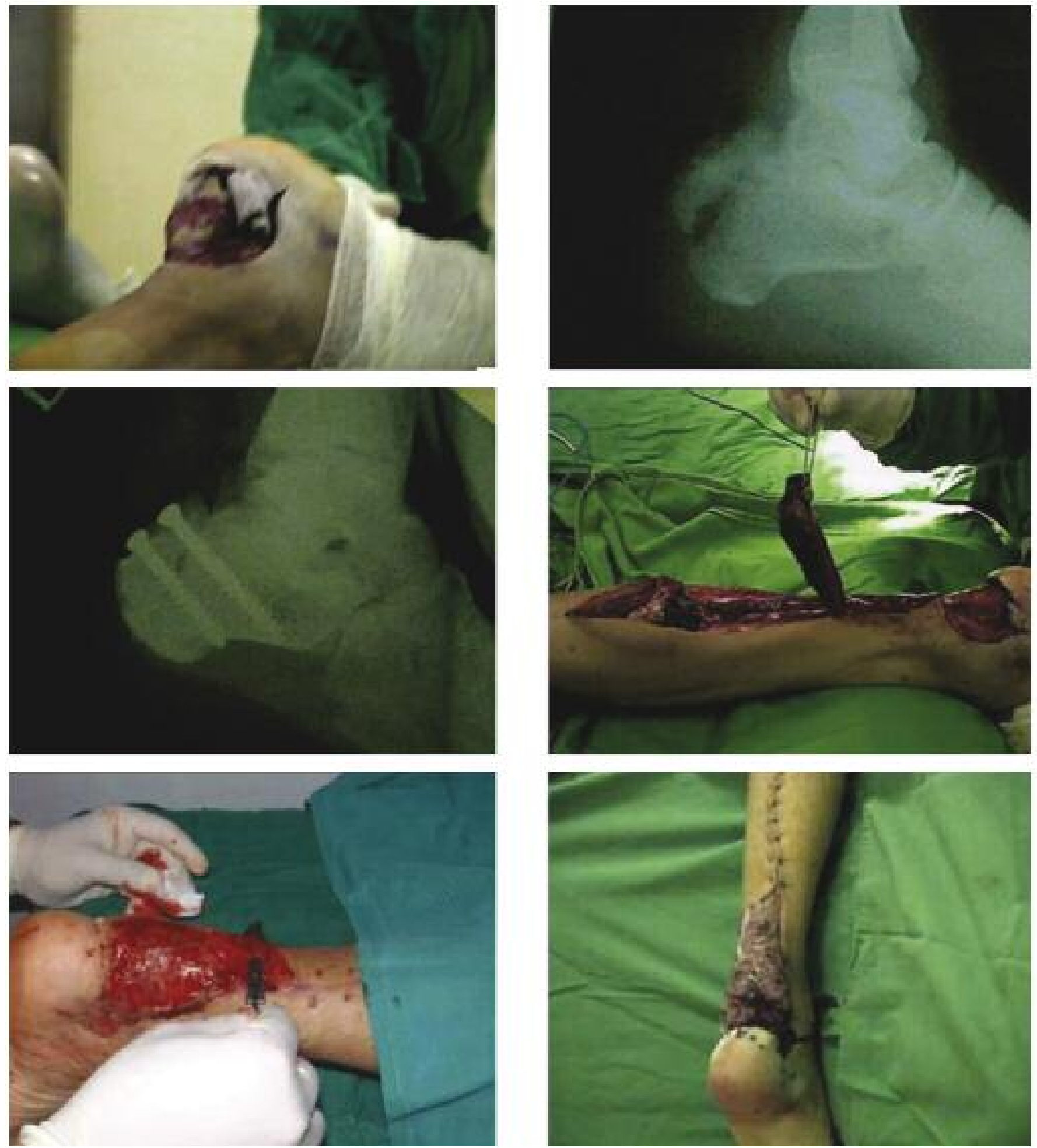

Fig. 4. Fractura de calcáneo izquierdo, grado III de Gustilo. Se realizó Colgajo de So, utilizando el cuadrante inferior externo del sóleo, rotado en ángulo de $180^{\circ}$; injerto de piel parcial a las tres semanas, e inmediatamente inicio de la rehabilitación.

No tuvimos ningún tipo de complicación ni con el colgajo ni con los injertos. El tiempo de hospitalización del paciente siempre dependió del tratamiento traumatológico.

En cuanto al inicio de la deambulación fue a los 12 y 15 días en los 2 primeros casos y en el tercer caso se inició al mes con la ayuda de muletas y a los 3 meses pisando firme.
Todos los casos fueron tratados simultáneamente con el Servicio de Traumatología.

De esta forma pudimos lograr una rehabilitación más rápida, porque la atrofia muscular y la granulación sobre el músculo, por ser de menor tamaño, se hace más rápidamente y permite al paciente deambular y calzarse más pronto. 


\section{Casos clínicos}

\section{Primer Caso}

Varón de 37 años de edad que sufrió accidente de tráfico. Presentaba fractura de tercio medio de tibia y peroné grado II de Gustilo. El defecto a reparar era una pérdida de sustancia de aproximadamente $5 \mathrm{~cm}$. de diámetro en el tercio superior de la región ántero-externa.

Para cubrir el defecto empleamos un colgajo del cuadrante ínfero-interno del músculo sóleo, tunelizado y fijado a la región anterior, con lo que el defecto cerró por completo. Realizamos el injerto a la tercera semana (Fig. 2).

\section{Segundo Caso}

Varón de 40 años de edad, que sufrió caída de moto. Como consecuencia, presentó fractura de tercio inferior de tibia y peroné grado II de Gustilo. El defecto a reparar se encontraba en tercio inferior, región ántero-externa y era muy irregular. Empleamos para su cobertura un colgajo de cuadrante ínfero-interno de músculo sóleo, tunelizado y fijado al defecto. A las 3 semanas se realizó el injerto de piel (Fig. 3).

\section{Tercer Caso}

Varón de 32 años de edad, que sufrió accidente de tráfico. Como consecuencia presentó fractura de calcáneo izquierdo grado III de Gustilo. Empleamos en este caso un colgajo de cudrante ínfero-externo de músculo sóleo, rotado en ángulo de 180 grados e injerto de piel parcial a las 3 semanas (Fig.4), momento en el que además, el paciente comenzó la rehabilitación.

\section{Conclusiones}

Observando los resultados estéticos y funcionales de los colgajos de músculo sóleo en otros pacientes operados con anterioridad, nos dimos cuenta de que resultaban un poco grotescos y algo molestos para el calzado y para los movimientos activos de los pacientes; es por esto que decidimos, basándonos en los criterios vasculares de los colgajos (9-10), realizar una variante que soportara la cobertura y la funcionalidad del miembro inferior.

Nuestra variante consiste en utilizar en vez de la mitad superior o inferior, sólo un cuarto del músculo sóleo, que según el caso puede ser el derecho o el izquierdo, superior o inferior, conservando sólo un pedículo vascular debido al ángulo de rotación que tuvimos que aplicar, y aunque la viabilidad de la mitad distal, según la literatura, es menos segura que la de la mitad proximal, la hemos utilizado con éxito.

$\mathrm{Al}$ realizar esta innovación hemos podido apreciar ventajas en los resultados, como por ejemplo el menor grosor del colgajo, sin abultamientos innecesarios que estéticamente incomodan al paciente e impiden a veces que se pueda calzar con normalidad.

Por otra parte la zona donante conserva mejor su forma natural y técnicamente el procedimiento se vuelve menos traumático al tener que disecar menos tejido. Por último, la recuperación funcional del miembro inferior se realiza en menor tiempo.

\section{Dirección del autor}

\author{
Dr. Carlos Márquez Zevallos \\ Hospital Clínica Alcívar \\ Coronel 2207 y Cañar \\ Primer Piso Consultorio 114. \\ Guayaquil, Ecuador. \\ e-mail.: dr_carlos_marquez@yahoo.com
}

\section{Bibliografía}

1. Vásconez, O.: "Colgajos Musculares y Musculocutáneos". Editorial Jims, Barcelona. 1984.

2. Mathes, S y Nahai, F: "Classification of the vascular anatomy of muscles: Experimental and clinical correlation". Plast and Reconst. Surg 1981, 67: 177

3. Gustilo R.B., Mendoza R.M.: "Problems in management of type III open fractures. A new classification of type III open fractures". J. Trauma 1984, 24:742.

4. Masquelet A. y col.: "Técnicas quirúrgicas. Los Colgajos musculares y cutáneos", Editorial Springer Verlag Ibérica S.A., Barcelona 1992, Pp. 45-57.

5. Godina M.: "Early microsurgical reconstruction of complex trauma of the extremities". Plast.Reconstr. Surg. 1986, 78: 285.

6. McCraw J.B.: "Selection of alternative local flaps in the leg and foot". Clin Plast. Surg., 1989, 6: 227.

7. Mathes S., Nahai F.: "Clinical Atlas of muscle and musculocutaneous flaps". Editorial Mosby Co., St.Louis, C. V.; 1982, Pp. 198203.

8. Townsend P. K. G.: "An inferiorly based Soleus muscle flap". Brit. J. Plast. Surg. 1988, 31: 210

9. Stark, W. J.: "The use of pedicled muscle flaps in the surgical treatment of compound fractures". J. Bone Joint Surg. 1996, 28: 343 .

10. Vasconez, L. y col.: "Coverage of exposed bone by muscle transposition and skin grafting”. Plast. Reconstr. Surg. 1994, 96: 526. 


\title{
Comentario al trabajo "Colgajo de So en reconstrucción de miembro inferiorı
}

\author{
Dr. Luis O. Vasconez \\ Profesor de Cirugía y Jefe de Cirugía Plástica. Universidad de Alabama. Birmingham, Al. (EE.UU.)
}

La trasposición de músculos locales para la cobertura de pérdidas de piel en la región de la tibia ha sido un adelanto importante. Hay suficiente experiencia clínica para indicar que los defectos en el tercio proximal de la tibia se pueden cubrir con la trasposición del gemelo (gastrocnemius) interno. Ocasionalmente, para defectos más laterales, podemos utilizar el gemelo lateral, con cuidado de no dañar el nervio peroneal que cruza alrededor de la cabeza del peroné.

Para defectos del tercio distal de la pierna, por lo general se aconseja el uso de un colgajo libre debido a que los colgajos locales tienen un riesgo muy alto. Ultimamente, se ha popularizado el uso de un colgajo fascio-cutáneo o neurocutáneo de base distal, tomado con su piel de debajo del área poplítea y basado en un pedículo vascular septocutáneo más bajo, que se localiza aproximadamente $5-6 \mathrm{~cm}$ por encima del maleolo (1).

Para defectos del tercio medio, se aconseja la trasposición del músculo soleo.

El uso de este músculo tiene ciertas dificultades:

1. Con frecuencia está involucrado en la fractura y en el trauma, y su arco de rotación es menor al expuesto en los libros. La razón es que cuando se describió dicho arco de rotación de los colgajos musculares, se hizo en base a disecciones en cadáveres frescos. Los músculos en cadáver son flácidos, no se contraen, no tienen cicatrices y por lo tanto, tienen un arco de rotación exagerado. Es fruto de esta observación el nacimiento de la advertencia "Todo el colgajo sobrevive, excepto lo que se necesita".

2. El músculo soleo es satisfactorio para la cobertura de defectos pequeños $(5 \mathrm{~cm})$ en el tercio medio de la pierna, particularmente si el cirujano obtiene la mayor longitud posible de músculo disecándo-

\section{Respuesta al comentario del Dr. Luis O. Vasconez}

\section{Dr. Carlos Márquez Zevallos}

Los comentarios vertidos por el Dr Luis Vascónez, son sumamente valiosos y dan las pautas para el uso de los colgajos musculares en la reconstrucción de miembro inferior de una manera didáctica y practica.

Mi experiencia con el injerto simultaneo en la rotación de otros colgajos musculares, no ha sido buena y en realidad no sé a qué atribuirla, pues en algunos casos el injerto se eliminó teniendo que recurrir a otro tiempo quirúrgico.

Dadas estas circunstancias no he querido arriesgar y, como son lo con bisturí de su inserción en la cara anterior del tendón de Aquiles. Para defectos mayores, puede fallar o tener un éxito parcial.

3. Debido a la experiencia menos que satisfactoria, y para hacer el colgajo más seguro, se han descrito modalidades que a veces hacen más daño que beneficio. Me refiero por ejemplo al uso del hemisoleo dividiendo una de las arterias principales de la pierna, distalmente a la trifurcación. Igualmente, el hemisoleo basado en los pedículos distales por lo general sufre necrosis, por lo menos parcialmente, o en el mejor de los casos produce un abultamiento excesivo.

Por las razones arriba indicadas, creo que la contribución y modificación de los Drs. Márquez et al. es efectiva, segura y útil para la cobertura de los defectos indicados.

Yo difiero con los autores en dos puntos relativamente pequeños. Uno: yo trato de injertar con un injerto de malla al mismo tiempo de la trasposición del músculo. Esto evita una segunda operación a las tres semanas como indican los autores. Segundo; yo planeo las incisiones como una extensión del defecto tanto proximal como distal, que ofrece suficiente acceso al soleo, y tiene la ventaja de que permite hacer la operación con el paciente en decúbito supino, lo que evita tener que tunelizar el músculo.

Recomiendo este artículo con la modificación indicada por la facilidad y seguridad de la técnica descrita. Debe ser incluida en las opciones abiertas para el cirujano plástico y reconstructivo.

\section{Bibliografía}

1. Follmar K.E., Baccarani A., Baumeister S.P., et al.: "The Distally Based Sural Flap". Plast. Reconstr. Surg. J. 2007, 119 (6): 138e. pacientes que por su problema traumatológico requieren de algún tiempo hospitalario, me he decidido por esperar hasta la atrofia muscular y el consiguiente crecimiento del tejido de granulación.

En cuanto al abordaje, me da una mejor visión panorámica que beneficia mi disección y sección del colgajo, dándome una medida exacta de lo que necesito para cubrir el defecto. Sin embargo, me parece interesante y lo intentaré para ver qué tal va en mis manos.

Finalmente agradezco la atención y sus comentarios. 